\title{
THE
}

\section{Wave Kinematics at High Sea States}

\author{
David Farmer \\ University of Rhode Island, farmerd@uri.edu
}

Li Ding

Donald Booth

Martin Lohmann

Follow this and additional works at: https://digitalcommons.uri.edu/gsofacpubs

\section{Citation/Publisher Attribution}

Farmer, D., Ding, L., Booth, D., \& Lohmann, M. (2002). Wave Kinematics at High Sea States. Journal of Atmospheric Oceanic Technology, 19, 225-239. doi: 10.1175/1520-0426(2002)0192.0.C0;2

Available at: https://doi.org/10.1175/1520-0426(2002)0192.0.C0;2

This Article is brought to you for free and open access by the Graduate School of Oceanography at DigitalCommons@URI. It has been accepted for inclusion in Graduate School of Oceanography Faculty Publications by an authorized administrator of DigitalCommons@URI. For more information, please contact digitalcommons-group@uri.edu. 


\title{
Wave Kinematics at High Sea States
}

\author{
DAVID FARMER \\ Graduate School of Oceanography, University of Rhode Island, Naragansett, Rhode Island
}

LI DING

Nexwave Inc., Baldwin Park, California

DONALD BOOTH

Autonetics Research Associates, Sooke, British Columbia, Canada

MARTIN LOHMANN

LICengineering A/S, Hellerup, Denmark

(Manuscript received 19 April 2000, in final form 20 March 2001)

\begin{abstract}
Measurements of currents close to the ocean surface and within the crests of large, steep waves have been acquired with an incoherent bistatic sonar mounted on the seafloor. The sonar uses a single narrow-beam transmitter/receiver and three fan-beam receivers set in a triangular configuration around the source. Acoustic pulses transmitted from the seafloor are scattered by bubble clouds and the sea surface to the four receivers and may be transformed into velocity components as a function of elevation. Individual estimates of the currents at, and close to, the surface are made with sufficient temporal resolution to identify kinematics in the crests of large waves. Observations acquired in the Danish sector of the North Sea are examined to evaluate both the potential merits and limitations of the measurement approach. At lower wind speeds, sidelobe scatter from the surface reaches the receiver simultaneously with the volume scattered signal arriving from a few meters beneath, contaminating the velocity measurement at this depth. At higher wind speeds, bubble clouds and increased roughness of the surface combine to suppress this effect, permitting reliable near-surface measurement. A numerical simulation has been implemented to explore some aspects of sonar performance including turbulent velocity fluctuations and bubble density gradients. Additional analysis is carried out to examine bubble suppression of sidelobe scatter. The observations lead to some conclusions regarding wave kinematics during a storm in which the wind speed reached $\sim 17 \mathrm{~m} \mathrm{~s}^{-1}$. At the ocean surface, the downwind velocity in the crests of large waves substantially exceeds that predicted by the second-order Stokes model, but in the wave troughs the current is close to the nonlinear prediction.
\end{abstract}

\section{Introduction}

Wave motion in large steep seas is important both to the understanding of wave dynamics and to maritime safety, naval architecture, and offshore engineering design. A particular challenge lies in the accurate measurement of wave properties at higher sea states. Surface elevation can be rather well measured using a variety of techniques including capacitance wire gauges, radar, and accelerometer wave buoys. Although sea surface elevation is of fundamental importance to wave studies, it is a quite specific type of information that can only

Corresponding author address: Dr. David Farmer, Graduate School of Oceanography, University of Rhode Island, Naragansett, RI 028821197.

E-mail: dfarmer@gso.uri.edu be converted into other variables such as flow velocity near the surface under certain assumptions. The validity of these assumptions may be open to question, especially in the case of large and steep waves. Here we describe an approach to the measurement of wave kinematics that exploits a bistatic Doppler sonar mounted on the seafloor.

The present work grew out of a practical need to measure flow speeds in extreme waves relevant to offshore engineering design (Dal 1997). A particular concern in this connection is the drag imposed by wave orbital motions on slender structures piercing the surface. The drag in turn depends on the square of the flow speed, which is a highly variable function of depth and time. Traditional calculations of this effect are based on a transformation of surface elevations into velocity time series using various approximations to the nonlinear ki- 
nematics, but uncertainty remains as to the validity of such an approach. Reliable estimates of wave kinematics require direct measurement of the three components of the current velocity profile in the upper few meters of the water column, together with the corresponding surface elevation. The required temporal resolution must be sufficient to resolve the flow field within individual waves and the measurements are most needed when the wave field is most extreme.

Given the violence of the ocean surface in severe weather, these requirements present a formidable challenge that is unlikely to be met with in situ measurement schemes. Accordingly, we selected a remote sensing approach using a bistatic configuration of sonars on the seafloor. The system was deployed in the North Sea close to a production platform in the Tyra field of the Danish sector in water of depth $\sim 38 \mathrm{~m}$. The present discussion describes the measurement concept and illustrates aspects of its operation with a mathematical model. The model calculations are then compared with observations.

\section{Sonar design and field operations}

Our approach involved the use of a bistatic multiple Doppler sonar system deployed on the seafloor. The use of bistatic systems is not new, having been developed with a different geometry for wind measurement with Doppler radars (Wurman et al. 1993). Coherent bistatic acoustic Doppler measurements of flow in the ocean have been made under the ice and in the surf zone ( $\mathrm{T}$. Stanton 2000, Naval Postgraduate School, personal communication). However, the application of incoherent technology to study the kinematics of ocean waves at high sea states extends the application in a new direction. The purpose of the present study is both to report on practical experience with this approach and to identify some of its possibilities and limitations.

The geometry is illustrated in Fig. 1 and includes a single narrow-beam source pointing straight upward, together with three fan-beam hydrophones in a triangular pattern arranged around the source. Pulses transmitted from the central sonar travel upward through the water column where they are scattered primarily by bubbles. The sound is scattered isotropically by the bubbles and is detected at four locations: the three surrounding hydrophones and the transmitting transducer, which also operates as a receiver. The fan-beam sonars were sensitive across $3^{\circ}$ in azimuth, but $\sim 60^{\circ}$ in elevation, allowing reception of sound from each point above the source. The $100-\mathrm{kHz}$ source used a circular transducer with $-3 \mathrm{~dB}$ beamwidth of $3.50^{\circ}$. The primary scattering signal of interest is due to bubbles distributed in the upper $\sim 10 \mathrm{~m}$ of the water column. Each receiver in the triangular array is horizontally separated from the source by $\sim 40 \mathrm{~m}$, which is also the total water depth.

The signals were detected and digitally transmitted via cable to a nearby oil production platform where they

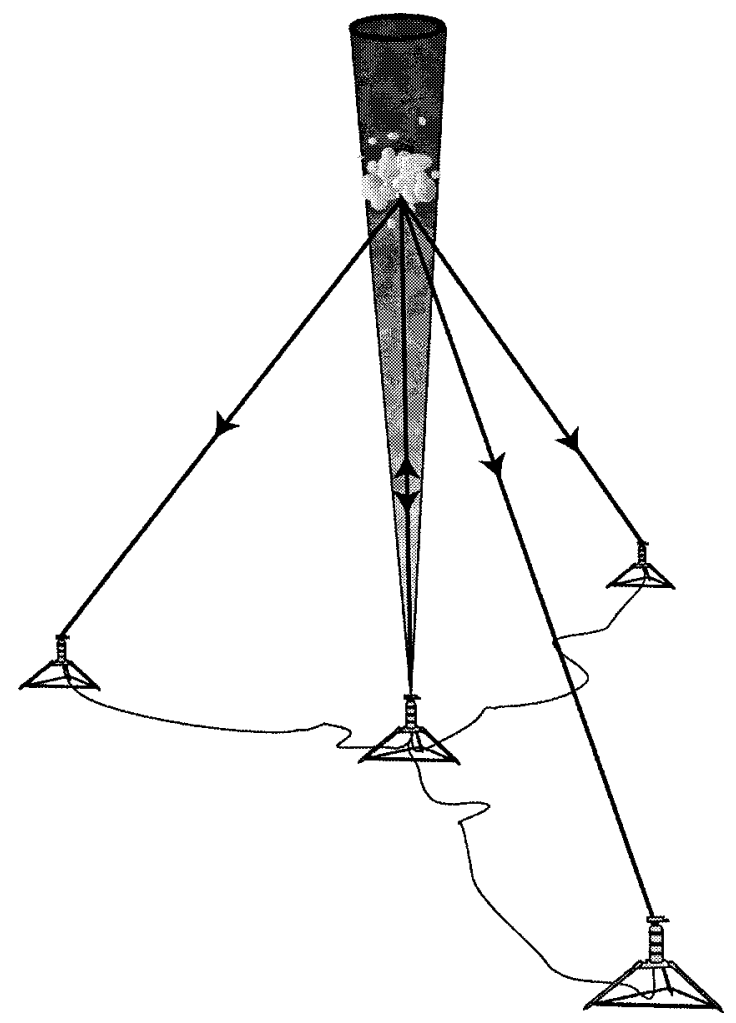

FIG. 1. Experimental setup. A narrow-beam source points up towards the surface. Fan-beam hydrophones detect the signal, which is obliquely scattered by bubbles and other targets in the water column. The source transducer also acts as a receiving hydrophone.

were stored on magnetic tape for subsequent processing. The use of narrow intersecting beams in a system that was deployed at four separate locations required some precautions to ensure correct alignment. Following deployment, transducer orientation was remotely controlled from the platform using a stepper motor and gear assembly at each seafloor site, permitting accurate alignment of the beams. The transducers were deployed close to the planned locations, but precise positions were determined acoustically.

The sonar operates at a nominal frequency of 100 $\mathrm{kHz}$ with $6 \times 5$ identical repeated Barker codes (Pinkel and Smith 1992; Trevorrow and Farmer 1992), each of duration $4.08 \mathrm{~ms}$ transmitted at a repetition rate of 3.62 $\mathrm{Hz}$. These provide an effective vertical resolution of $3.26 \mathrm{~m}$, representing a compromise between desired velocity and range resolution. The sonar electronic design is identical to that used in quite different backscatter systems for battery-powered operation on drifting buoys (Farmer and Li 1995). Following detection, the signal is mixed to $3675 \mathrm{~Hz}$, demodulated, and recorded on magnetic tape with the Pulse Code Modulation Scheme. The sonar is tuned for backscatter from the clouds of bubbles that fill the upper few meters of the ocean at higher wind speeds. Thus the acoustic targets are located in just that part of the water column in which our interest 


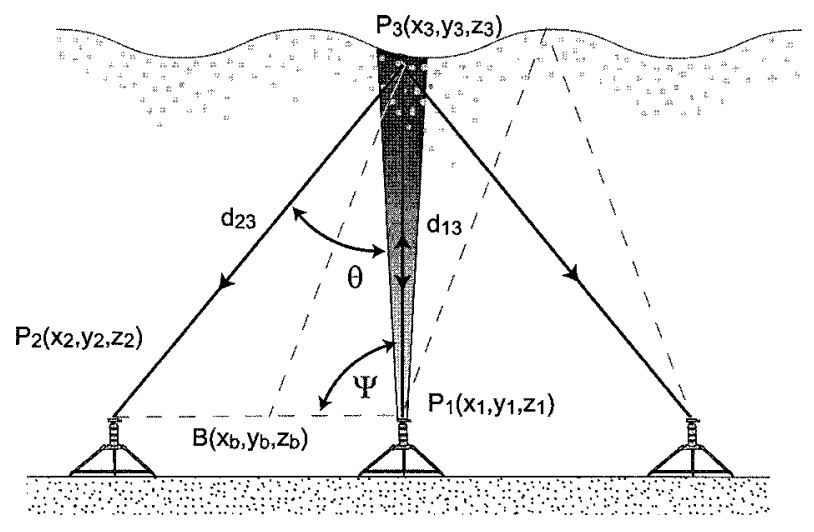

FIG. 2. Sideview of bistatic configuration. The dashed triangular path intersecting the surface to the right indicates the source of potential sidelobe contamination from the sea surface.

in the currents is greatest and they provide an excellent signal-to-noise ratio. In the absence of bubbles, the surface is easily recognizable as a sharp jump in backscatter target strength. However, from time to time at the highest wind speeds, the bubble density can be great enough to make the surface hard to identify, necessitating development of a different signal processing approach discussed below.

Practical constraints precluded our physical presence on the platform for the measurement period, but one of the platform personnel was able to switch the system on from time to time so that data were recorded; however, no tuning or orientation adjustments were possible. This introduced a problem when one of the tripods began to settle leading to a slight misalignment. It was found that data were still recoverable, however, and the limitations were to some degree overcome by exploiting the redundancy afforded by a four sonar measurement system designed for resolving three velocity components.

\section{Measurement principles}

Consider a bistatic setup as in Fig. 2, where $\mathrm{P}_{1}$ is the transmitter, $\mathrm{P}_{2}$ a receiver and, $\mathrm{P}_{3}$ a moving target. A typical ray from $\mathrm{P}_{1}$ is scattered isotropically by $\mathrm{P}_{3}$ so that a signal is received at $\mathrm{P}_{2}$ as shown, where $\angle \mathrm{P}_{1} \mathrm{P}_{3} \mathrm{P}_{2}$ $=\theta$. It can be easily shown that for a transmitted signal with a wavelength of $\lambda_{0}$, the Doppler frequency shift resulting from the moving target is given by

$$
\Delta f=-\frac{1}{\lambda_{0}} \mathbf{v} \cdot\left(\mathbf{r}_{1}+\mathbf{r}_{2}\right),
$$

where $\mathbf{v}$ is the velocity vector, and $\mathbf{r}_{1}, \mathbf{r}_{2}$ are the unit vectors from the transmitter and receiver to the target, respectively. Since $\left|\mathbf{r}_{1}\right|=\left|\mathbf{r}_{2}\right|=1$, it can be shown

$$
\Delta f=-\frac{2}{\lambda_{0}} \cos \left(\frac{\theta}{2}\right) v_{b},
$$

where $v_{b}$ is the velocity component along $\mathrm{P}_{3} B$, the bisector of $\theta$.

The Doppler frequency shift can be derived from received signals using a complex covariance approach (Miller and Rochwarger 1972). Let $Q(t)$ and $I(t)$ represent the quadrature and in-phase components of the received signals, which can be obtained from the realtime series with a quadrature demodulation technique. The covariance is defined as

$$
R(\tau)=\sum_{n=1}^{N} Z^{*}\left(t_{n}+\tau\right) Z\left(t_{n}\right),
$$

where $Z(t)=Q(t)+i I(t)$. The Doppler frequency shift is then estimated as

$$
\Delta f=-\frac{1}{2 \pi \tau} \arg \{R(\tau)\},
$$

where arg denotes the phase of a complex number. The time lag $\tau$ is set to be the code length $\left(0.68 \times 10^{-3} \mathrm{~s}\right)$ of transmitted signals, leading to a maximum unambiguous velocity of about $5.7 \mathrm{~m} \mathrm{~s}^{-1}$ for the system.

Equation (2) indicates that if $\Delta f$ can be measured, the velocity component along the bisector direction can be determined. Referring to Fig. 1, the scattered sound can be received at four locations. It is possible to reconstruct the three-dimensional velocity from the bisector velocity component measured at these locations, by choosing an appropriate coordinate and evaluating the geometric relationship between the transmitter, the receivers, and the target. The mathematical equations are given in appendix A.

\section{Sources of error in Doppler velocity measurements}

\section{a. Random phase noise}

A fundamental limit on the performance of an incoherent Doppler system is the Cramer-Rao (CR) lower bound (Theriault 1986), which, for a code of $L$ bits, repeated $M$ times in each transmission, is given by (Pinkel and Smith 1992) for the case of large signal-to-noise ratios:

$$
\sigma_{v}^{2} \geq \frac{3}{2}\left(\frac{\lambda}{4 \pi}\right)^{2} \frac{1}{L^{3}(M-1)^{2} \tau_{b}^{2}},
$$

where $\sigma_{v}^{2}$ is the velocity variance and $\tau_{b}$ the bit length. It is assumed that the standard time-domain covariance method is used with the time lag $L \tau_{b}$ and the averaging period $(M-1) L \tau_{b}$. For our present system, the lower bound derived from the error inherent in the covariance estimate is $0.19 \mathrm{~m} \mathrm{~s}^{-1}$ for velocity measurements from the transducer and $0.20 \mathrm{~m} \mathrm{~s}^{-1}$ for those from the sidescan sonars.

The actual velocity variance of a system is larger than the CR bound, due to ambient and electronic noise, random motion of scatterers and other factors. The sig- 
nal-to-noise ratio (SNR) at the receivers depends on the transmitted source level, bubble density, and ambient noise levels. For a fixed source level, the echo strength increases with wind speed due to an increase in bubble density, but ambient noise levels also increase. By examining received data at various wind speeds, we find that the source level is sufficient to ensure a high SNR and thus the effect of ambient and electronic noise is small. Random motion of scatterers leads to spectral spreading and thus additional velocity variance, as discussed by Cabrera et al. (1987) and Brumley et al. (1991). They identify three individual contributions: residence time of scatterers in an insonified volume, beam divergence, and turbulence. Following their analysis, we find that the largest contribution comes from near-surface turbulence. Recent field measurements suggest that turbulent dissipation rates near the surface are up to two orders of magnitude higher than wall theory predicts (Agrawal et al. 1992; Drennan et al. 1996). Based on these results we estimate that at a wind speed of $17 \mathrm{~m} \mathrm{~s}^{-1}$ and depth of $1 \mathrm{~m}$, the dissipation rate is $\varepsilon$ $=3.3$ and the resulting Doppler spectral bandwidth is $B=39 \mathrm{~Hz}$, corresponding to a decorrelation time $\tau_{d}=$ $1 /(2 \pi B)=4.1 \times 10^{-3} \mathrm{~s}$. The time lag used in the covariance approach is $0.68 \mathrm{~ms}$, which is much smaller than the decorrelation time. Therefore, the impact of random motion is expected to be small.

The bubble density in the near-surface environment decreases rapidly with depth. The bubble density typically decays approximately exponentially with an averaged $e$-folding depth of $\sim 1 \mathrm{~m}$ at moderate wind speeds. Since the 3-m spatial resolution of our system could be significantly greater than the typical $e$-folding depth of bubble density distributions, the backscatter from bubbles within the same range cell will be spatially inhomogeneous, leading to additional spectral spreading. In order to assess the effect, we have developed a numerical simulation model, taking due account of real system and environmental parameters. We choose an appropriately large number of bubbles satisfying a radius distribution range consistent with observations. The positions of the bubbles are determined using a random number generator subject to a given vertical distribution function. In general, each bubble will move under the combined effects of turbulence, buoyancy, mean flow, and wave motion, which leads to a corresponding Doppler shift $\delta c$ for each bubble. These combine to form a random variable in the summation over all bubbles and will lead to spectral broadening. In the model we simulate the source signal so as to be consistent with the instrument's coding scheme. The amplitude and phase of the insonifying signal at each bubble and its echo are determined by the position of the bubble relative to the transmitter and receiver. Attenuation factors due to spherical spreading and excess extinction within the bubbly medium are also included. A Doppler frequency shift is introduced for each bubble based on its velocity and total received signals are the summation of all the echoes. The model reproduces the signal processing carried in the real data analysis and the resulting simulated Doppler shifts are extracted. The details of the simulation can be found in appendix B.

We simulated received signals for both a vertically uniform and an exponential distribution of bubbles, with the $e$-folding depth determined by experimental data described in appendix B. Representative wave orbital velocity fields were used. It is found that the resulting velocity deviation for a single pulse scattered by a uniform bubble distribution is $0.21 \mathrm{~m} \mathrm{~s}^{-1}$ at the obliquely oriented hydrophones. For an exponential bubble distribution of $e$-folding depth $1 \mathrm{~m}$, the simulation predicts an increase in velocity deviation of $0.3 \mathrm{~m} \mathrm{~s}^{-1}$. The simulation confirms that under the aforementioned dissipation rate, the effect of turbulence on the signal is insignificant. The acoustical signal scattered by the sea surface is generally very well defined for this geometry and the resulting Doppler predicitions appear to be robust. However, Doppler measurement beneath the surface is subject to weighting by the variable bubble concentration, leaving some room for ambiguity, since the resolution in bubble distribution and in velocity measurement is limited by the transmit pulse duration. Further exploration of this issue and the development of improved signal processing and analysis techniques remains the subject of investigation (M. Lohmann 2000, LICengineering and Danish Technical University, personal communication).

\section{b. Signal contamination from surface scatter}

Our measurement principle relies on the assumption that signals received at the obliquely oriented sonars are dominated by scatter from a common scattering volume. However, the signals may be contaminated by unwanted echoes. Signal contamination resulting from multiple scattering and sidelobe coupling is recognized as one of the drawbacks of the bistatic Doppler radar system described in Wurman et al. (1993). This problem is usually negligible with monostatic transmitting narrowbeam radars, but can be significant in the bistatic system due to the low gains and wide viewing angles of the receivers. While we do not expect the scenarios described in Wurman et al. to be significant in our application, a more serious problem is the proximity of the sea surface. The transmitting sonar does not have an ideal "pencil" beam and although narrow in azimuth, the obliquely oriented hydrophones have a beam width of $60^{\circ}$ in elevation; thus, sound traveling along the sidelobes can be reflected at the surface and arrive at the sidescan hydrophones. The surface is a strong acoustic reflector, thus raising a concern that the reflections may contaminate the volume scatter of interest, especially when the bubble density is low. It is therefore necessary to estimate the potential effects of sidelobe reflections.

The theory of sound scattering from a random rough surface has been an active subject of research (Ogilvy 
1992; Medwin and Clay 1998). Application to our experiment is particularly complicated as the data of interest were collected at high states where the mean wave height was much larger than the acoustic wavelength; thus, multiple reflections and shadowing may have occurred. However, as a first step toward estimating the surface scatter contamination, we neglect these secondorder effects, even though they may be significant, and follow the simple approach described in Medwin and Clay (1998). Specifically, we estimate the specular surface scattering strength. We then compare this estimate with the bistatic scattering strength of the bubble clouds, taking into account attenuation due to bubbles. Detailed calculations are given in appendix $\mathrm{C}$.

Our analysis focuses on the strongest sidelobe at a beam angle of $15^{\circ}$. Specular reflections due to the sidelobe arrive at the receiver simultaneously with bistatic scatter from bubbles about $3 \mathrm{~m}$ below the surface. For the lowest sea state $\left(W=7.3 \mathrm{~m} \mathrm{~s}^{-1}\right)$, the sidelobe reflection is $38 \mathrm{~dB}$ stronger than the bistatic scatter from bubble clouds, completely dominating the bubble scatter. In this case, we cannot obtain any meaningful measurements of current velocity at 3-m depth. On the other hand, at the highest wind conditions encountered ( $W=$ $17 \mathrm{~m} \mathrm{~s}^{-1}$ ), the bubble scatter is $21 \mathrm{~dB}$ stronger than the sidelobe and reliable velocity measurements are predicted. Thus our analysis predicts that sidelobe reflection would seriously contaminate velocity measurements at 3-m depth at low sea states but the effect would be avoided at high sea states. As discussed subsequently, this prediction is borne out in the data analysis. At intermediate sea states, the effect is more difficult to assess, since the sidelobe reflection would be of similar magnitude to that of bistatic scatter from bubble clouds, requiring more accurate modeling and bubble measurements that lie beyond the scope of the present paper. However, we note here that our motivation for the study was to test the validity of assumptions relating surface elevation to near surface velocities. Such a test assumes its greatest significance in extreme conditions; it is in these conditions, where the bubble scatter is strongest, that near surface velocity measurement can be achieved.

\section{Data analysis}

The Doppler system was deployed from October 1994 to January 1995 at the Tyra East platform complex in the Danish sector of the North Sea (Fig. 3). The North Sea is relatively shallow with a water depth of around $38 \mathrm{~m}$ and a relatively flat, horizontal seafloor at the experimental site. The combination of a larger wind fetch for northwest winds and the limited water depth gives rise to a severe winter wave climate with frequent occurrence of steep breaking waves.

During the deployment period, the acoustic system was activated 10 times, covering different sea states from calm seas, with a wave height of only $2 \mathrm{~m}$, to severe storms with wave heights exceeding $8 \mathrm{~m}$. Here

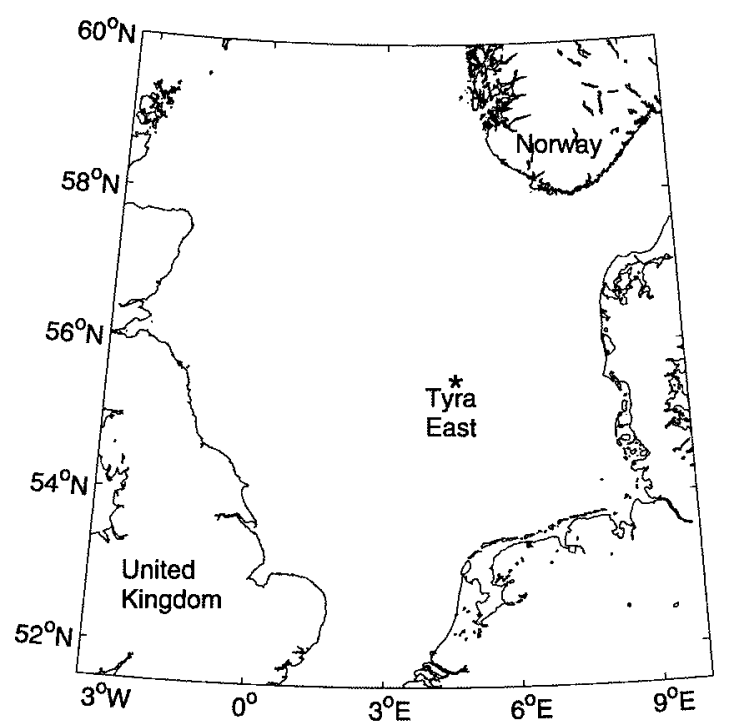

FIG. 3. Location of the experimental site. The measurements were acquired in water of $\sim 38 \mathrm{~m}$ depth near a production platform in the Tyra East field of the Danish sector.

we select two datasets for analysis. One of these was from the most severe storm (wind speed $17 \mathrm{~m} \mathrm{~s}^{-1}$ and significant wave height $8.2 \mathrm{~m}$ ) and demonstrates the ability of the system to measure extreme wave velocities. The other is from relatively calm conditions (wind speed $7.3 \mathrm{~m} \mathrm{~s}^{-1}$ and significant wave height $1.4 \mathrm{~m}$ ) and illustrates the effect of sidelobe reflections from the sea surface.

\section{a. Determination of surface elevation}

The instantaneous surface elevation can be measured from the signal detected by the vertically oriented transducer. A surface echo has a range dependence of $1 /(2 h)$, where $h$ is the distance from the surface to the transmitter; a common method of surface range estimation makes use of an amplitude threshold to detect surface echoes. The presence of dense bubble clouds just beneath the surface can blur the surface signature. We detected the change in signal associated with reflection from the sea surface of the end of the transmitted pulse and found that use of a time variable gain led to cleaner wave height measurements.

The surface echoes usually far exceed any volume scatter, thus ensuring clear detection of the surface. However, in extreme sea states, acoustic scatter from bubble clouds can sometimes be comparable to the surface echo, which is also reduced through bubble attenuation, causing the simple threshold surface detection method to fail. One example is shown in Fig. 4a, where the apparent surface elevation measured from threshold detection drops below mean surface level for the period 200-250 s, due to dense bubble clouds penetrating to a depth of $10 \mathrm{~m}$. This leads to anomalies in the measurement of wave height. For this $\sim 4$ h dataset, dropouts 

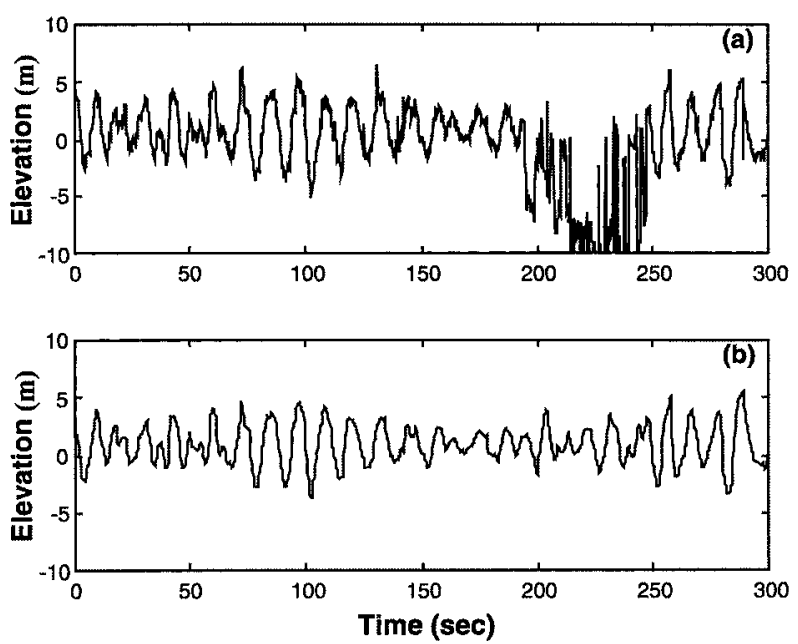

FIG. 4. Acoustically determined surface elevation obtained from the vertically oriented sonar over a 5-min period at high sea state. (a) Measurement based on the threshold method, illustrating dropout due to dense bubble clouds. (b) The result obtained by integrating the vertical velocity data.

of this magnitude occurred six times, each event lasting 3-4 min. We therefore adopt a different approach, in which the vertical velocity measured from the vertical Doppler sonar at the presumed surface location, is integrated to obtain a new estimate of surface elevation. This is a reasonable step since the first-order derivative of surface elevation should be close to the vertical velocity. The calculation is repeated iteratively, although convergence is rapid. We show the result in Fig. 4b, from which it is seen that the anomalous dropouts are removed.

\section{b. Surface and subsurface velocities}

After filtering the data in this way, the surface elevation is used as the position reference to calculate surface and subsurface velocities. We first examine the vertical velocity $V z$, which can be obtained either directly from the vertically oriented transmitter-receiver, or indirectly from the three obliquely oriented hydrophones (see appendix A). Inconsistency in the results can be expected if the signal is contaminated by sidelobe scatter from the surface.

Figure 5 shows a comparison of $V z$ obtained with the two methods, at the surface, and at 3 and $6 \mathrm{~m}$ below the instantaneous surface, for the high and low sea state. The correlation coefficient between velocity estimates by both methods is also shown in the figure. In general, there is no significant difference between estimates of the vertical speed at the ocean surface, whether measurements are made at high or low sea states, since the surface echo immediately above the transmitter arrives earlier than the sidelobe reflection and remains uncontaminated. For the subsurface velocities in the low sea state, however, it can be seen that the estimates differ greatly, especially at 3-
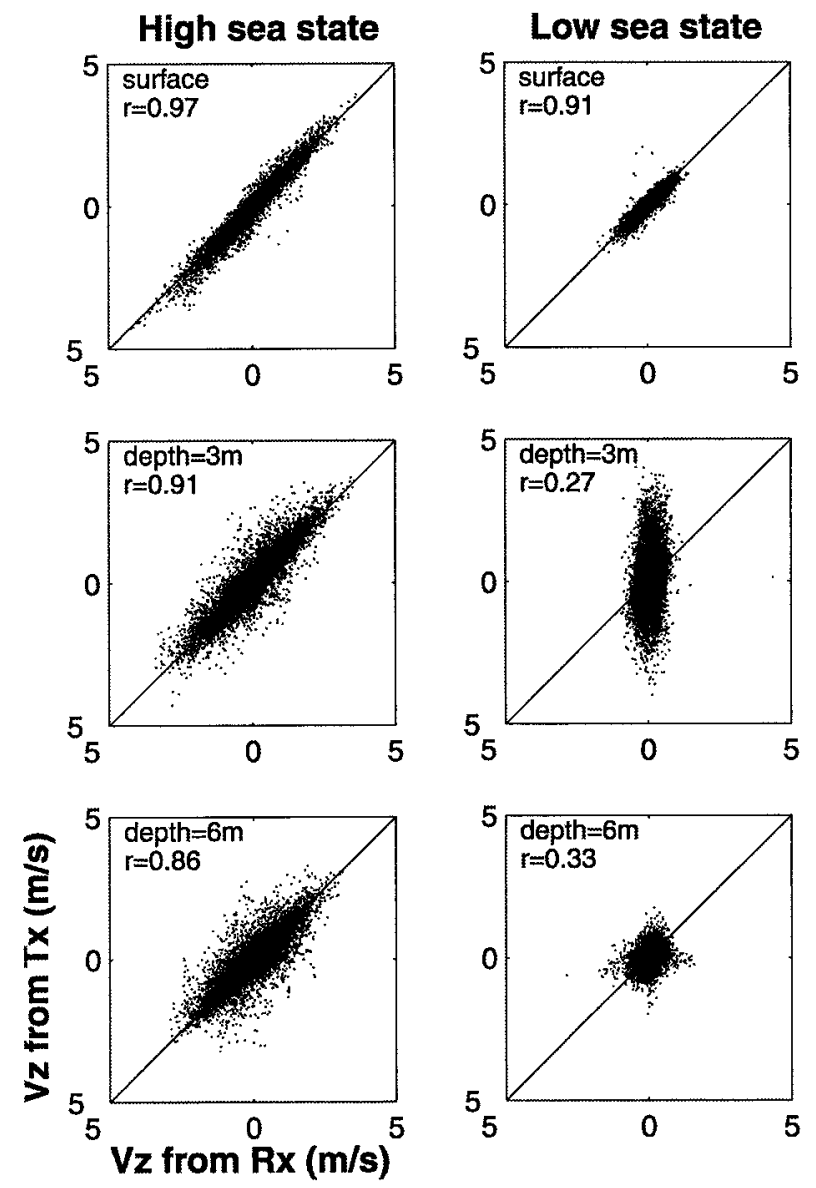

FIG. 5. Comparison of vertical velocity derived from a single vertical Doppler beam ( $V_{z}$ from $R_{x}$ ) and vertical velocity derived from the signals acquired only with the bistatic receivers $\left(V_{z}\right.$ from $\left.T_{x}\right)$. Each data point represents an average over $30 \mathrm{~min}$. The measurements made using two different approaches are consistent at high sea states, but inconsistent at low sea states due to sidelobe interference.

$m$ depth. Nevertheless, in the high sea state example, the two estimates at 3-m depth are essentially identical, with a correlation coefficient of 0.91 , indicating that sidelobe reflections are not significant. These results are consistent with our conclusion that although sidelobe contamination is serious at low wind speeds, it is masked by the bubble scatter and attenuation at high wind speeds. At 6-m depth, the vertical velocity estimates from the sidescan hydrophones are again consistent with those derived from the transmitter, but with a reduced correlation coefficient (0.86). The reduced correlation may be due to the increased thickness of the ensonified cell with depth (see appendix C).

We also calculated the received backscatter intensity profile (as a function of depth) for the transmitter and sidescans. If the backscatter comes from the same scattering volume, all the backscatter profiles should have similar shapes. If sidelobe interference exists, the profiles for the obliquely oriented hydrophones will show increased intensity at some depth. We have examined 

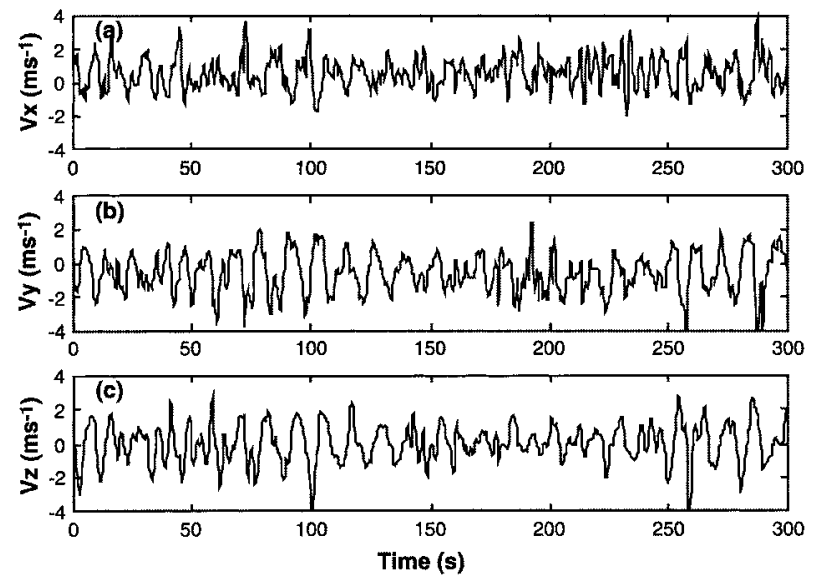

FIG. 6. Time series of three velocity components (a) $V_{x}$, (b) $V_{y}$, and (c) $V_{z}$, at the surface, derived from the bistatic configuration at high sea state $\left(U_{10} \sim 17 \mathrm{~m} \mathrm{~s}^{-1}\right)$.

the profiles in various sea states and found that at low and intermediate sea states $\left(U_{10}<15 \mathrm{~m} \mathrm{~s}^{-1}\right)$, the backscatter does indeed increase over the range 3-6 m beneath the surface as predicted for sidelobe contamination. This effect disappears at high sea states.

On the basis of our examination of vertical velocity time series at various depths, we decided that it would be more consistent to maintain the same ensonified volume and calculate the components of velocity from the three obliquely oriented receivers. The $-3 \mathrm{~dB}$ ensonified surface area has a surface footprint of $2.2 \mathrm{~m}$. The highest spatial resolution is therefore $2.2 \mathrm{~m}$ so that the minimum unambiguously resolvable wavelength is 4.4 $\mathrm{m}$. This leads to a maximum measurable wave frequency of $0.6 \mathrm{~Hz}$. We therefore apply a low-pass filter with 0.6 $\mathrm{Hz}$ cutoff frequency to the velocity data, which are then inverted to obtain the three velocity components $V x, V y$, and $V z$.

Figure 6 shows a time series of $V x, V y$, and $V z$ at the surface for the high sea state data, and Fig. 7 shows the results at depth $9 \mathrm{~m}$. The 9-m velocities are attenuated but in phase with the surface measurements shown in Fig. 6. The resulting horizontal surface and subsurface velocities have a nonzero mean $\left(m V x=0.53 \mathrm{~m} \mathrm{~s}^{-1}\right.$ and $m V y=-0.62 \mathrm{~m} \mathrm{~s}^{-1}$ at the surface; $m V x=0.30 \mathrm{~m} \mathrm{~s}^{-1}$ and $m V y=-0.41 \mathrm{~m} \mathrm{~s}^{-1}$ at $9-\mathrm{m}$ depth). This is primarily due to two factors: near-surface shear and wave nonlinearities. Recent observations show that close to the surface the turbulent diffusivity is substantially enhanced relative to "law-of-the-wall" scaling in a wind driven sea (Gemmrich and Farmer 1999), but we expect vertical shear in the upper few meters. Currents within the mixed layer can result from tides, wind-driven currents, and geostrophic flow. Wave nonlinearities tend to enhance particle orbital velocities near wave crests and reduce them near troughs, but the effect can be averaged out over time. We use the time-averaged horizontal ve-
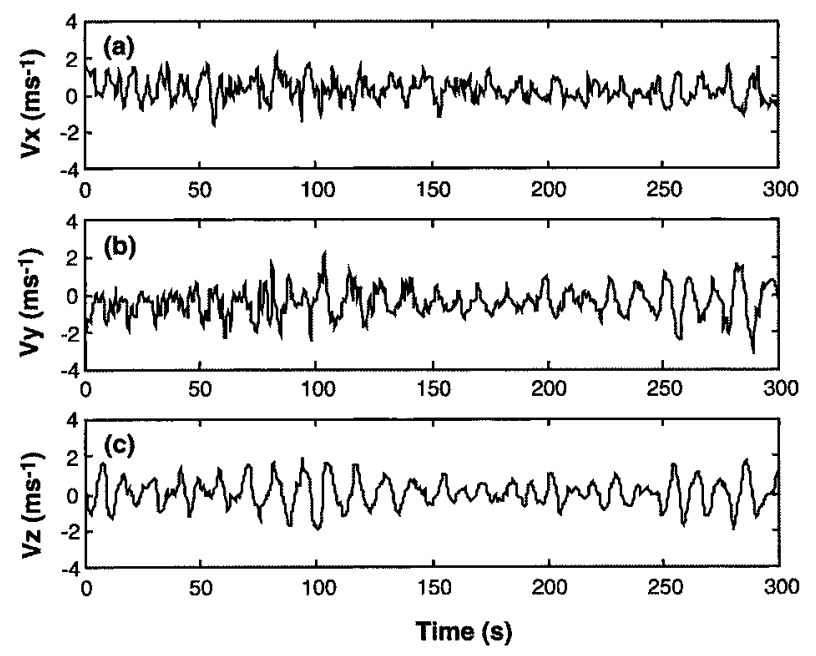

FIG. 7. Three velocity components at 9-m depth derived from the bistatic configuration at high sea state $\left(U_{10} \sim 17 \mathrm{~m} \mathrm{~s}^{-1}\right)$.

locity at $9 \mathrm{~m}$ as an estimate of the residual mixed layer current and subtract it from the velocity data.

\section{c. Interpretation with an irregular linear wave model}

We have determined that the sonar data provide reasonably accurate measurements of vertical surface velocity. However, from the point of view of wave kinematics, the horizontal components are of greater interest. Linear wave theory provides a useful starting point for examining the results. Less steep waves can be expected to approximate this theory; significant deviations for more extreme waves should stand out and are of intrinsic interest. Based only on two-dimensional deep water linear theory, for a monotonic, progressive wave, the displacement $\eta(x, t)=A \cos (k x-\omega t)$, vertical velocity $w$, and horizontal velocity $u$ at the mean water level $z=0$ are given by

$$
w=\frac{\partial \eta}{\partial t}, \quad u=\operatorname{coth}(k H) \frac{\partial \tilde{\eta}}{\partial t},
$$

where $k$ is the wavenumber and $H$ is the water depth. The term $\tilde{\eta}$ is the Hilbert transform of $\tilde{\eta}$, defined as

$$
\tilde{\eta}(t)=\pi^{-1} \int_{-\infty}^{\infty} \frac{\eta(t)}{t-\xi} d \xi
$$

For analysis of narrowband wave fields, $\eta$ is replaced by surface elevation data; $w$ and $u$ may then be derived from the first-order derivative of the surface elevation and its Hilbert transform. If the wave data are broad band and the water is not deep, the results need to be expressed in terms of Fourier transforms to take account of the factor $\operatorname{coth}(k H)$. This simple model assumes that both the dynamical and kinematic boundary conditions are linear. It may, however, implicitly include nonlinearity if measured surface elevation already shows some nonlinearity. 

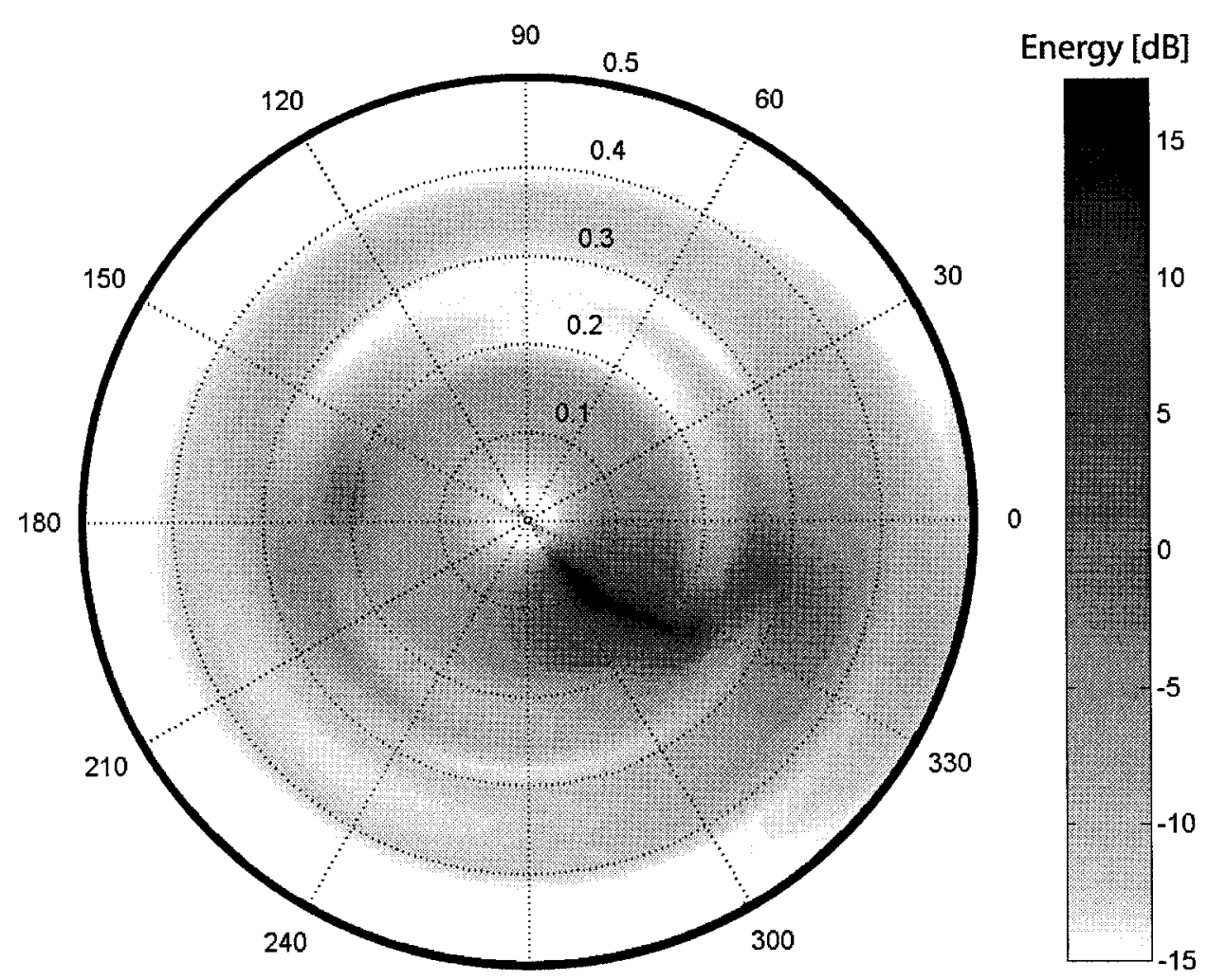

270

FIG. 8. Directional wave spectrum calculated by the maximum entropy approach for the high sea state data analyzed. The power spectrum grayscale has the units $\left(\mathrm{m} \mathrm{s}^{-1}\right)^{2} \mathrm{~Hz}^{-1}$ expressed in decibels.

We use this model to examine our measurements of horizontal orbital velocity. Since the measurements were made in a $3 \mathrm{D}$ wave field and the model is two-dimensional, we can only compare the model with the data
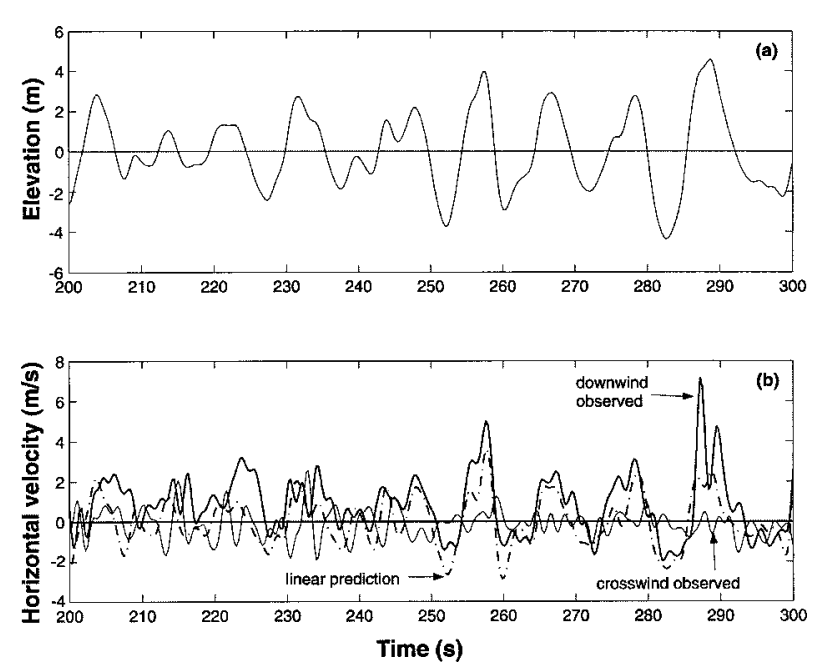

FIG. 9. Comparison between the measured downwind horizontal velocity at the surface and the irregular linear model prediction $\left(U_{10}\right.$ $\sim 17 \mathrm{~m} \mathrm{~s}^{-1}$ ): (a) surface elevation and (b) horizontal velocity. The measured cross-wind velocity is also shown. in the dominant wave direction. We first determine the wave direction from the 3D velocity data using the maximum entropy approach described in Lygre and Krogstad (1986) and then, for the observed surface elevation, compare the measured horizontal velocity component in the wave direction with the model prediction.

For the measured velocity time series, the directional wave spectrum determined with the maximum entropy method indicates a dominant wave component of period $\sim 10 \mathrm{~s}$ traveling toward $150^{\circ} \mathrm{N}$ (Fig. 8). The maximum value of $\operatorname{coth}(k H)$ in $(6)$ is $1.07(H=38 \mathrm{~m})$, and approximated as unity. The horizontal velocity data are rotated into the dominant wave direction and the resulting component along the wave direction compared with (6). Figure 9 shows such a comparison for a short period encompassing a large steep wave. The upper figure shows the observed surface elevation and the lower shows the corresponding horizontal component of measured velocity resolved in the wave direction, together with the model prediction (dotted line). The elevation time series used in the prediction is derived from the vertically oriented sonar and is independent of the signals detected on the obliquely oriented hydrophones from which the horizontal velocity is derived. The model prediction is largely consistent with the data except for the anomalously large and steep wave at $\sim 285 \mathrm{~s}$. 


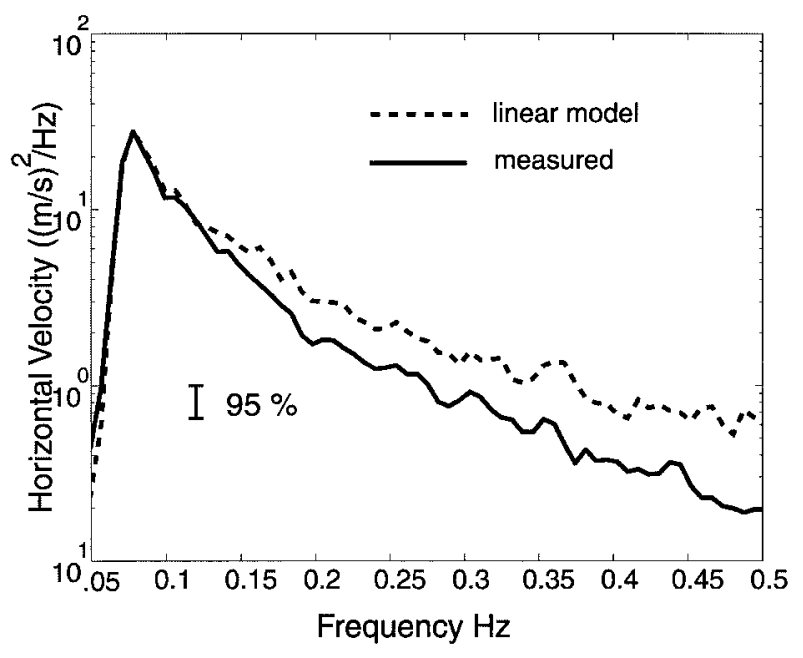

FIG. 10. Power spectrum of measured downwind surface velocity and linear model prediction. At higher wavenumbers the linear prediction does not accommodate directional spreading and overestimates the wave spectrum.

The velocity near the crest of this wave reaches $7 \mathrm{~m}$ $\mathrm{s}^{-1}$ while the linear model predicts a value less than 5 $\mathrm{m} \mathrm{s}^{-1}$. These features are representative of other large and steep waves in the time series.

Figure 10 shows the power spectra of measured horizontal velocities and corresponding linear predictions. The linear theory coincides with the data at frequencies below $0.12 \mathrm{~Hz}$, but overestimates the results at higher frequencies. The excess velocities evident for large steep waves (i.e., at $\sim 285 \mathrm{~s}$ in Fig. 9) are relatively infrequent and unlikely to influence significantly the time-averaged power spectrum. This overall discrepancy in the spectrum at higher frequencies is to be expected as the linear theory has been applied to a two-dimensional approximation of the observations; the real wave field becomes progressively more three-dimensional at higher frequencies and is underestimated in our representation. This does not affect our subsequent analysis in which we focus on properties of individual large, steep waves.

\section{d. Orbital velocity at the wave crest}

Figure 9 shows that the orbital velocity near the wave crest can exceed the linear prediction by more than $40 \%$. Before extending the analysis to larger datasets, we explore the potential of second-order nonlinear theory to explain the difference. Random waves can be described by the energy spectrum, but can also be considered a succession of discrete waves, each of which has its own wave period and amplitude. There are a number of criteria that define "discrete waves" in a time series of surface elevation. The "zero-up-crossing" (ZUC) and "zero-down-crossing" (ZDC) criteria are commonly used; a wave is defined by two consecutive ZUCs or ZDCs. An alternative approach is the crest-to-crest criterion in which a wave is defined by the passage of two consecutive maxima in the surface elevation. One disadvantage of these criteria is that if there are a significant number of small waves, either real or false waves may arise from measurement errors and the resulting amplitude or period statistics will be biased toward the smaller scales. Therefore, in practice, a threshold is often used to eliminate some of the small waves (Pires-Silva and Medina 1994). One can also apply a low-pass filter to surface elevation data before extracting discrete waves from the time series. The choice of a threshold or a cutoff frequency for the low-pass filter can be quite subjective.

Another criterion has recently been proposed by $\mathrm{Pi}$ res-Silva and Medina (1994) and Gimenez et al. (1994), which defines a wave as a $2 \pi$ advance in the phase of the complex function:

$$
A F(t)=\eta(t)+i \tilde{\eta}(t)
$$

The advantage of this approach, referred to as the orbital criterion, is that it is relatively insensitive to interference caused by random noise, since a small disturbance is unlikely to change the overall phase by $2 \pi$. It therefore eliminates the need to choose a threshold or use a lowpass filter. It would be more likely to ignore small waves riding on long waves. However, since our analysis focuses on large or extreme waves, the orbital criterion is more suitable for our data analysis.

With this criterion we can extract individual waves and their parameters. For example, the wave period is defined as the time interval between $2 \pi$ phase changes (in practice, when the phase is changed by more than $1.5 \pi$, a wave is defined) and wave height is defined as the vertical distance from the local minimum to maximum within the wave period. A wave crest is defined as the time at which the surface elevation is maximum within a wave period. The wave parameters derived in this way can then be used in appropriate models to predict the crest velocity for comparison with the corresponding data.

We now reexamine linear theory and some classical nonlinear wave theories, and compare them with the wave data. First, we note that linear theory yields a particle velocity profile relative to a mean water level. The velocity at the instantaneous surface is considered the same as that at the mean water level. We can use the Taylor expansion to obtain velocity profiles relative to the instantaneous surface (Dean and Dalrymple 1984):

$$
u\left(x, \eta+z_{0}\right)=u\left(x, z_{0}\right)+\left.\eta \frac{\partial u}{\partial z}\right|_{z=z_{0}},
$$

where $\eta$ is the surface displacement at the mean water level $z_{0}=0$, and $z$ is depth relative to the mean level. If we assume a single progressive wave $\eta(x, t)=A$ $\cos (k x-\omega t)$, we find 


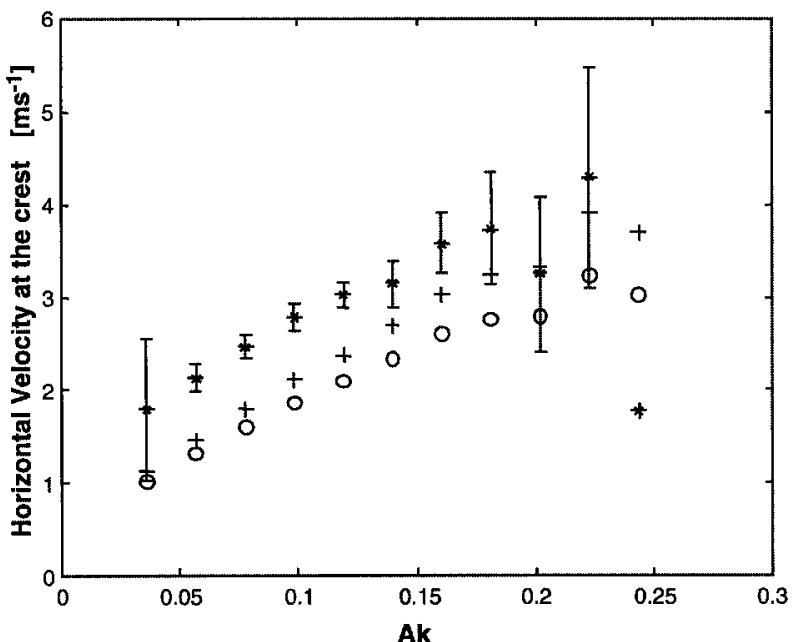

FIG. 11. Comparison of near-crest horizontal velocity measured at the surface with theoretical predictions. Only waves exceeding 4-m range are included. The asterisks (*) represent data, the open circles (O) represent the linear model using the Taylor expansion, and the plus sign $(+)$ denotes the second-order Stokes nonlinear prediction. The data are averaged results (over individual waves in a 4-h period), and the $90 \%$ confidence interval is also shown except for the highest value, for which only one wave was observed.

$$
\begin{aligned}
u(x, & \left.\eta+z_{0}\right) \\
= & \frac{g A k}{\omega} \frac{\cosh \left[k\left(H+z_{0}\right)\right]}{\cosh (k H)} \cos (k x-\omega t) \\
& +\frac{g(A k)^{2}}{\omega} \frac{\sinh \left[k\left(H+z_{0}\right)\right]}{\cosh (k H)} \cos ^{2}(k x-\omega t),
\end{aligned}
$$

where $H$ is the water depth. At the surface, (10) predicts the magnitude of horizontal velocity is increased at the crest and decreased at the trough. The resulting surface horizontal velocity has a nonzero average value. Unlike the Stokes theory discussed below, (10) calculates velocity at the instantaneous surface, consistent with our Doppler velocity estimates derived from surface acoustical scatter.

Next consider the second-order Stokes expansion, for which the boundary conditions are nonlinear. If the firstorder (linear) wave has the form $\eta_{0}(x, t)=A \cos (k x-$ $\omega t)$, the surface elevation and horizontal velocity, to second order, are (Dean and Dalrymple 1984):

$$
\begin{aligned}
\eta(x, t)= & A \cos (k x-\omega t) \\
& +\frac{1}{4} A^{2} k \frac{\cosh (k H)}{\sinh ^{3}(k H)}[2+\cosh (2 k H)] \\
& \times \cos 2(k x-\omega t), \text { and } \\
u\left(x, z_{0}\right)= & \frac{g A k}{\omega} \frac{\cosh k\left(H+z_{0}\right)}{\cosh (k H)} \cos (k x-\omega t) \\
& +\frac{3}{4} \frac{g(A k)^{2}}{\omega} \frac{\tanh (k H) \cosh 2 k\left(H+z_{0}\right)}{\sinh ^{4}(k H)} \\
& \times \cos 2(k x-\omega t) .
\end{aligned}
$$

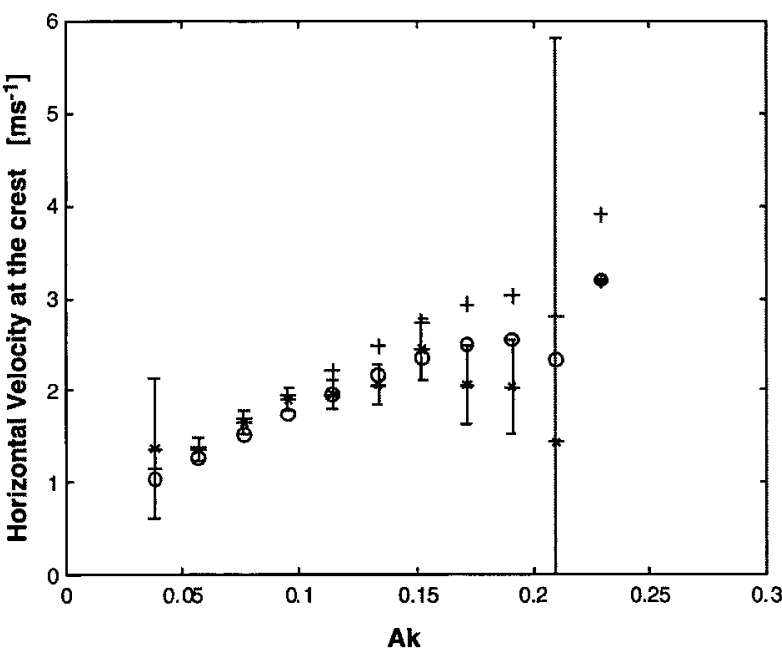

FIG. 12. Comparison of near-crest horizontal velocity corresponding to Fig. 11 measured at 3-m depth with two wave models. Symbols, data averaging, and confidence limits are as in Fig. 11.

The presence of the second-order term increases the velocity at the crest, but in contrast to the Taylor expansion solution (10) its effect is averaged out over time. We can also substitute (12) into (9) to obtain the effect of second order nonlinearity on horizontal velocity relative to the instantaneous surface.

The analysis of discrete waves provides us with individual wave parameters, such as wave period and height, with which to test the applicability of the secondorder Stokes prediction at the instantaneous surface. Individual wave data of amplitude $A$ and wavenumber $k$ are binned according to slope $A k$; the wavenumber $k$ is found from the dispersion relation and measured wave period. We impose a constraint that the angle between the surface velocity vectors at the crest and trough be greater than $135^{\circ}$ and that the wave height be greater than $4 \mathrm{~m}$. This ensures that the wave parameters are associated with the same wave and limits our analysis to large waves. Substituting the mean wave number for each bin into (9)-(12), we calculate linear and nonlinear predictions of horizontal velocity at the crest. We also find the mean measured velocity at the crest for individual waves falling in each bin. Figures 11 and 12 show the results at the surface and 3-m depth, where the data are represented by asterisks, the linear predictions by circles, and second-order Stokes predictions by plus symbols. It is seen that the measured horizontal velocities at the crests are consistently larger than the theoretical predictions, although the second-order prediction does better at higher slopes. For $A k>0.2$ the observations are two sparse for consistent comparison. In contrast, the linear and nonlinear predictions at $3 \mathrm{~m}$ lie within the $90 \%$ confidence bounds of the data for slopes up to 0.15 . Figure 13 shows the comparison for horizontal velocities at the surface, conditionally sampled at the wave trough; over most of the measured range, 


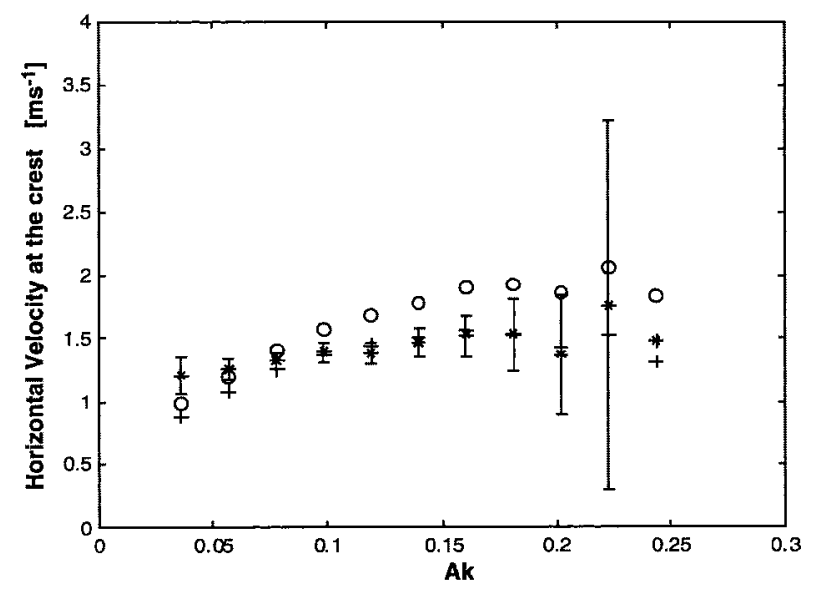

FIG. 13. Comparison of horizontal velocity measured at the surface close to the troughs of large waves $(4 \mathrm{~m})$ corresponding to Fig. 11. Symbols, data averaging, and confidence limits are as in Fig. 11.

the observations generally correspond to the secondorder predictions but are overestimated by the linear model.

Higher-order calculations, such as the fifth-order Stokes expansion or the streamfunction theory are often used in offshore engineering design. In the present case, application of the streamfunction theory has been applied (N. E. Ottesen-Hansen 2000, LICengineering, personal communication), but it was found that for a slope of $0.15-0.20$, the prediction was only $10 \%-15 \%$ greater than the linear prediction. These results indicate that existing theories may underestimate velocities at, or very close to, the surface within the crests of large steep waves. On the other hand, as shown in Fig. 12, the predictions appear to provide an adequate description of wave kinematics at depths greater than a few meters, even in severe sea states.

\section{Summary}

We have described a bistatic Doppler sonar system for measuring surface wave orbital motion and presented preliminary measurements of surface velocities at higher sea states acquired in the North Sea. The bistatic system was able to acquire reliable measurements of the three velocity components at the ocean surface. The ability of the sonar to measure the horizontal components of velocity in the range bin located just beneath the surface is limited at low and moderate sea states by interference from sidelobe reflections. However, at high sea states, which are of particular interest with respect to the study of surface wave kinematics, the dense bubble layer beneath the surface effectively attenuates sidelobe reflections and subsurface velocities appear to be unaffected.

The wave measurement concept presented here differs from commonly used acoustical methods, which employ monostatic acoustic Doppler current profiler technology. Alternatively, a local measurement of $u, v$, and $w$ at one fixed depth is also used. The more usual monostatic Doppler measurement makes use of beams that diverge from a single location; the accuracy of velocity estimates from this configuration must therefore depend on assumptions of horizontal homogeneity of the wave velocity field. This constrains the application of monostatic systems to wave fields for which the dispersion relation may be safely relied upon to recover the wave field. This is a reasonable first-order approximation except for large steep waves. The present approach is specifically intended for investigation of the near-surface environment at higher sea states, for which breakdown of linear and the classical nonlinear predictions of the near surface velocity field might be anticipated. Both numerical simulation and analytical investigation identifies factors influencing the accuracy of the measurement approach. We found that near-surface turbulence would have little impact on the wave measurements, despite high dissipation rates. Contamination of the Doppler signal detected a few meters beneath the surface by sidelobe reflections from the surface are a significant source of error at lower wind speeds, but are masked by higher bubble concentrations at higher sea states.

Velocity measurements of the kind presented here are required for accurate description of wave kinematics at high sea states and have practical applications such as wave drag prediction in offshore engineering design. In order to determine whether the data are consistent with existing wave theories, we have compared our observations with a second-order Stokes wave theory, using a wave-to-wave analysis. Comparison of observed and predicted horizontal velocities at high sea states $\left(U_{10}=\right.$ $17 \mathrm{~m} \mathrm{~s}^{-1}$ ) suggests that the linear and second-order Stokes models underestimate surface speeds at or near the crests of large, steep waves. However, wave kinematics at depths greater than a few meters is well approximated by linear theory.

Our experience with an incoherent bistatic sonar for near-surface measurements suggests a number of modifications that would make for improved results in a future implementation. Increased bandwidth and higherrange resolution would reduce the sensitivity to steep gradients in the bubble concentration and lead to lower variance in the measured velocity. In fact the present system was not optimized in this respect and a threefold increase in bandwidth is readily obtainable. The sidescan transducers that serve as hydrophones can also be used as transmitters. The resulting Doppler signal would then provide a basis for directional wave measurement.

By far the greatest concern, however, is sidelobe interference just beneath the surface. Some gains could be achieved by using a vertical sonar of greater aperture and shading to attenuate the sidelobes, but a better approach might be to use electronically steered beams on the obliquely oriented hydrophones. For example, replacement of each obliquely oriented hydrophone with 
a dipole pair, each channel of which is independently recorded, would allow selective steering of the dipole so as to place the receiver null at the point of sidelobe scatter, thus minimizing the potential for this kind of interference.

Acknowledgments. We gratefully acknowledge the contributions of many individuals and the financial support and other assistance provided by the several organizations involved in this project. Financial support was provided in Denmark through the Energy Research Program EFP-91 and EFP-94 by the Danish Ministry of Energy and in Canada by the National Energy Board and the Office of Energy Research and Development (Marine Transportation and Safety, Project 534202). The operator Mærsk Olie og Gas AS and DUC partners A. P. Moeller, Shell, and Texaco kindly assisted by providing access to the Tyra East platform and by installing instrumentation at sea. The Institute of Hydrodynamics and Hydrology, Technical University of Denmark contributed resources under the program "Marine Technique" funded by the Danish Technical Research Council (STVF). Dr. Eric Dal was responsible for the initial analysis of the North Sea data. We also acknowledge several helpful suggestions from our referees.

\section{APPENDIX A}

\section{Determination of 3D Velocity}

Referring to Fig. 2, we use a rectangular Cartesian coordinate system in which we have $\mathrm{P}_{1}\left(x_{1}, y_{1}, z_{1}\right), \mathrm{P}_{2}\left(x_{2}\right.$, $\left.y_{2}, z_{2}\right)$, and $\mathrm{P}_{3}\left(x_{3}, y_{3}, z_{3}\right)$. Let $B\left(x_{b}, y_{b}, z_{b}\right)$ be the intersection of the bisector of $\angle \mathrm{P}_{1} \mathrm{P}_{3} \mathrm{P}_{2}$ with $\mathrm{P}_{1} \mathrm{P}_{2}$. If $\alpha_{1}$, $\beta_{1}, \gamma_{1}$ and $\alpha_{2}, \beta_{2}, \gamma_{2}$ are the angles between $\mathrm{P}_{1} \mathrm{P}_{3}, \mathrm{P}_{2} \mathrm{P}_{3}$ and the $X, Y, Z$ axes, respectively, then the direction cosines of these lines are given by

$$
\begin{array}{ll}
\cos \alpha_{1}=\frac{x_{3}-x_{1}}{d_{13}}, & \cos \beta_{1}=\frac{y_{3}-y_{1}}{d_{13}}, \\
\cos \gamma_{1}=\frac{z_{3}-z_{1}}{d_{13}} & \\
\cos \alpha_{2}=\frac{x_{3}-x_{2}}{d_{23}}, & \cos \beta_{2}=\frac{y_{3}-y_{2}}{d_{23}}, \\
\cos \gamma_{2}=\frac{z_{3}-z_{2}}{d_{23}}, &
\end{array}
$$

where $d_{13}$ and $d_{23}$ are the lengths of $\mathrm{P}_{1} \mathrm{P}_{3}$ and $\mathrm{P}_{2} \mathrm{P}_{3}$. Furthermore,

$$
\begin{aligned}
\cos \theta= & \cos \alpha_{1} \cos \alpha_{2}+\cos \beta_{1} \cos \beta_{2} \\
& +\cos \gamma_{1} \cos \gamma_{2} .
\end{aligned}
$$

Let $\angle \mathrm{P}_{2} \mathrm{P}_{1} \mathrm{P}_{3}=\psi$ and $d x=\mathrm{P}_{1} B$. Then,

$$
d x=\frac{d_{31} \sin \theta / 2}{\sin (\theta / 2+\psi)}, \quad \cos \psi=\frac{d_{23}^{2}-d_{12}^{2}-d_{13}^{2}}{-2 d_{12} d_{13}} .
$$

The coordinates of $B$ are then given by

$$
\begin{aligned}
& x_{b}=x_{1}+\frac{d x}{d_{12}}\left(x_{2}-x_{1}\right), \quad y_{b}=y_{1}+\frac{d x}{d_{12}}\left(y_{2}-y_{1}\right), \\
& z_{b}=z_{1}+\frac{d x}{d_{12}}\left(z_{2}-z_{1}\right) .
\end{aligned}
$$

The direction cosines of $\mathrm{BP}_{3}$ are

$$
\begin{aligned}
& \cos \alpha_{3}=\frac{x_{3}-x_{b}}{d_{B P_{3}}}, \quad \cos \beta_{3}=\frac{y_{3}-y_{b}}{d_{B P_{3}}}, \\
& \cos \gamma_{3}=\frac{z_{3}-z_{b}}{d_{B P_{3}}} .
\end{aligned}
$$

Let $\mathbf{v}=\left\{v_{x}, v_{y}, v_{z}\right\}$ be the velocity vector of the target. Its component along $B P_{3}$ is given by

$$
v_{b}=v_{x} \cos \alpha_{3}+v_{y} \cos \beta_{3}+v_{z} \cos \gamma_{3} .
$$

Equation (A7) can be derived for each of the receivers, giving the flow velocity along the bisectors of the lines joining the mean position of the bubble to the origin and to the receivers. The 3D velocity components can be obtained by solving these equations for $v_{x}, v_{y}$, $v_{z}$.

\section{APPENDIX B}

\section{Numerical Simulation of the Bistatic Sonar}

Consider first the bubble field. We note that the single scatter approximation of a cloud of bubbles may be treated either as a continuous distribution with specified statistical properties or as a set of $n$ individuals each of which has a defined radius $r_{i}$ and a defined velocity vector of magnitude $v_{i}, i=1, \ldots, N$. Here we adopt the latter approach and choose an appropriately large number of bubbles satisfying a radius distribution range consistent with observations. The positions of the bubbles are determined using a random number generator, modified so that bubbles always lie within the ensonified volume and in a band of specified width immediately below the surface or according to some other specified distribution.

The bubble radius distribution determines the volume scattering coefficient and attenuation factors as a function of frequency. Since the bubble distribution was not measured during the experiment, we use direct measurements obtained with acoustic resonators near the ocean surface in the Gulf of Mexico (Farmer et al. 1998). The data were collected at five different depths with the wind speed ranging from 7.5 to $15 \mathrm{~m} \mathrm{~s}^{-1}$. Five data files were available at depths of 0.7, 1.3, 1.9, 3.3, and $5.5 \mathrm{~m}$. Bubble densities for a fixed wind speed were examined as a function of bubble radius.

The data fit the following empirical relation:

$$
n(a, z, W)=5.6 \times 10^{5} W \exp (-z / D) p(a),
$$

with 


$$
p(a)= \begin{cases}a^{-p_{1}} & a \leq a_{0} \\ \beta a^{-p_{2}} & a>a_{0},\end{cases}
$$

where $n(a)$ is the number of bubbles per cubic meter per micron radius increment, $W$ is wind speed in meters per second at $10 \mathrm{~m}$, and $z$ is depth below the instantaneous ocean surface. The $e$-folding depth $D$ is $0.7 \mathrm{~m}$, but the reader should be cautioned that our shallowest measurement was $0.7 \mathrm{~m}$. We found that $a_{0}=100 \mu \mathrm{m}$ was appropriate for the dataset, with $p_{1} \sim 1.75$ and $p_{2}$ $\sim 5.0$. The parameter $\beta=a_{0}^{\left(p_{2}-p_{1}\right)}=a_{0}^{3.25}$ is adjusted to ensure continuity at $a_{0}$. It should be emphasized that this is a simplification. For example, there is some evidence that $a_{0}$ decreases somewhat with depth and this is not included. There is also systematic evolution of the bubble size spectrum with time following injection by the breaking wave, which of course is not incorporated in this time averaged representation. Development of more comprehensive empirical representations based on more extensive data sets is the subject of ongoing work. This distribution was used for evaluating sidelobe interaction with the surface (appendix C), but for rapid simulation with large numbers of bubbles, we used a simpler expression suitable for the smaller bubbles most relevant to the sonar measurements described here:

$$
N(a)=4094 W \exp [1.111(z-0.7)-0.0409 a],
$$

where $W$ is the wind speed in meters per second, $z$ is the depth in meters, and $a$ is bubble radius in micrometers. The linear dependence on $W$ represents a simplification that will be relaxed as further data are acquired.

Buoyant rise of the bubbles is also a function of their radius. However, the high quality factor of bubbles at resonance ensures that over some range of frequencies the acoustic target strength may be assumed to arise primarily from resonant scattering and thus be limited to a narrow radius spectrum. Although we retain generality in the calculations by permitting a realistic range of radii and corresponding scattering cross section, for the experimental variables of interest here we are concerned with a narrow spectrum corresponding to the $100-\mathrm{kHz}$ sonar, centered at $30.7 \mu \mathrm{m}$, having a rise rate of $0.02 \mathrm{~m} \mathrm{~s}^{-1}$, which is negligible compared to wave and turbulence velocities and may be neglected. Turbulence is represented by a small random element added to each velocity component. This random element is assumed to be Gaussian distributed with its variance equal to the velocity variance within the inertial subrange of the turbulent spectrum. The dissipation rate was chosen to be consistent with recent field measurements (Agrawal et al. 1992; Drennan et al. 1996). Bubble motion was further modulated to include wave orbital velocities representative of the observations.

The transmitted wave form is assumed to be $\cos 2 \pi f t$ and to have unit amplitude, where $t$ is the time in sec- onds and $f$ the frequency in hertz. The incident wave at each bubble has a phase and amplitude determined by the position of the bubble relative to the transmitter. Attenuation is included for spherical spreading and excess attenuation in a bubbly medium. The echo at a receiver is then determined from the scattering function, the position relative to the receiver and the attenuation factors. Assuming attenuation factors $a_{1}\left(\mathbf{d}_{i}\right)$ and $a_{2}\left(\mathbf{r}_{i}\right)$, where $\mathbf{d}_{i}$ and $\mathbf{r}_{i}$ are the vectors from source to the target and from the target to the receiver, the average signal at the receiver from all bubbles within a given rangegated scattering volume is given by

$S=\frac{1}{N} \sum_{i=1}^{i=N} F_{i} a_{1}\left(\mathbf{d}_{i}\right) a_{2}\left(\mathbf{r}_{i}\right) \cos \left[2 \pi\left(f+\delta_{c}\right) t-\phi_{i}\right]$,

where

$$
\phi_{i}=\frac{2 \pi}{c_{0}}\left[f\left(d_{i}+r_{i}\right)+\delta_{c} r_{i}\right]
$$

and $F_{i}$ is the scattering function of the bubble. The attenuation factors implicitly include the transmitting and receiving beam pattern. The calculation compensates for motion of the bubble over the period of the transmitted pulse. Finally, the signal simulation at the hydrophone is analyzed following a procedure identical to that used in the real data analysis.

\section{APPENDIX C}

\section{Analysis of Sidelobe Reflections}

Kirchhoff theory is widely used to model acoustical scattering from a sea surface with large wave height. Medwin and Clay (1998) provide a derivation of scattering by rough surfaces, based on Kirchhoff theory, which incorporates effects due to finite transmitting and reception beamwidths. Generally, the surface scattering intensity can be expressed as

$$
\left\langle p_{\mathrm{ss}}^{2}\right\rangle=P_{0}^{2} R_{0}^{2} \frac{A S}{R_{1}^{2} R_{2}^{2}} b_{2}\left(\Theta_{1}, \Phi_{1}\right) \mathrm{Att}_{1},
$$

where $S$ is the surface scattering coefficient; $A$ is the effective ensonified area on the surface; and $R_{1}$ and $R_{2}$ are the distances from the ensonified center to the transmitter and receiver, respectively (see Fig. C1). The term $b_{2}\left(\Theta_{1}, \Phi_{1}\right)$ is the intensity directivity function of the receiver, $\mathrm{Att}_{1}$ is the attenuation factor, and $P_{0}$ is the source pressure at range $R_{0}=1 \mathrm{~m}$. It is assumed that there is no surface shadowing and that the ensonified area is small compared to $R_{1}$ and $R_{2}$.

The surface scattering coefficient is a complicated function of sea surface wave characteristics and the acoustic system parameters. In order to make the problem mathematically tractable, Medwin and Clay assume a Gaussian beam for the transmitter, for which the effective ensonified area is 


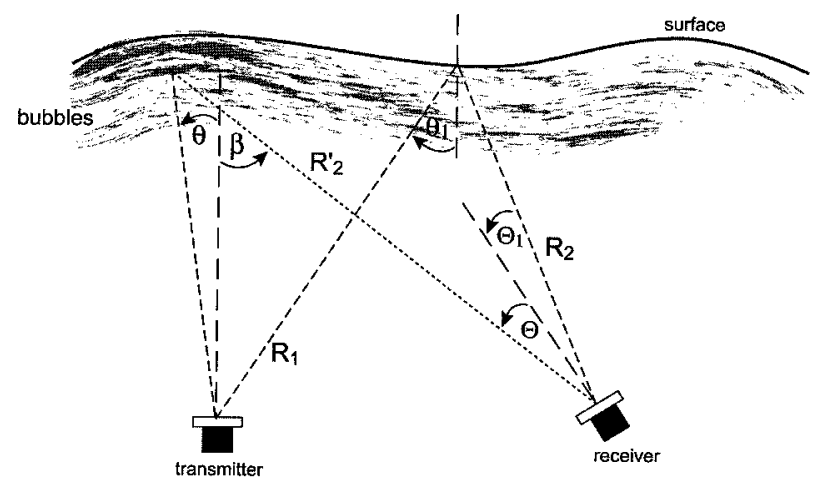

FIG. C1. Specular surface scatter, showing nomenclature used in analysis.

$$
A=\frac{\pi R_{1}^{2} \sin ^{2} \theta_{0}}{\cos \theta_{1}},
$$

where $\theta_{0}$ is the $e^{-1}$ pressure half beam angle and $\theta_{1}$ the incident angle. The calculation also requires knowledge of the spatial correlation function in the wave field, which can be obtained from detailed measurements of the directional wave spectra (Dahl 1999). Here we assume a quadratic form of the spatial correlation function for mathematical convenience.

A further simplification is based on properties of the transmitting and receiving beam patterns. The most significant sidelobe of the source transducer occurs at $\theta=$ $15^{\circ}$. Although the first sidelobe is at $\theta=6^{\circ}$, this has a weaker response and the angle is too small for specular reflections to be directed toward the obliquely oriented sonars. Therefore, we consider only the sidelobe at $\theta=$ $15^{\circ}$. The obliquely oriented sonar has a fan beam that is very broad in elevation $\left(\sim 60^{\circ}\right)$ and narrow in azimuth $\left(\sim 3^{\circ}\right)$. Each fan-beam hydrophone is directed in azimuth toward the transducer, so we need only consider surface reflections in the fan-beam plane and specular direction of the sidelobe. Although the obliquely oriented hydrophones do not point in the specular direction when the surface is flat, a moderate surface slope $\left(\sim 10^{\circ}\right)$ would be sufficient to direct sidelobe reflections toward the hydrophone in the specular direction. An estimate in the specular direction would give us an upper bound of the sidelobe effect.

Under these conditions, it can be shown that when the acoustic roughness,

$$
g=4 k^{2} \sigma_{h}^{2} \cos ^{2} \theta_{1},
$$

is large, the specular scatter asymptotically approaches

$$
S=\frac{C^{2}}{16 \pi \sigma_{s}^{2}} .
$$

This applies under the far-field condition

$$
\frac{R_{1}}{R_{2}} \ll \frac{4 \sigma_{s} \cos \theta_{1}}{\sin \theta_{0}}-1,
$$

where $\sigma_{h}$ and $\sigma_{s}$ are the rms surface displacement and surface slope and $C$ is the reflection coefficient for a perfectly smooth surface, taken as -1 in our application.

The sidelobe is estimated to have an equivalent width $\theta_{0}$ of $\sim 2^{\circ}$. The rms surface slope can be estimated from direct measurements of wave height (Cox and Munk 1954) and is found to vary from 0.17 to 0.26 for our data. Therefore the above far-field condition is satisfied in our application.

We now consider bistatic scatter from bubble clouds, which arrives at the obliquely oriented hydrophones at the same time as the sidelobe reflection. For the present configuration such scatter would come from bubbles $\sim 3$ $\mathrm{m}$ below the surface. Extending the Medwin and Clay's (1998) derivation to the bistatic configuration, we find

$$
\begin{aligned}
\left\langle p_{\text {bi }}^{2}\right\rangle= & P_{0}^{2} R_{0}^{2} \operatorname{Att}_{2} \frac{S_{v} \Delta R}{R_{2}^{\prime 2}} \\
& \times \int b_{1}(\theta, \phi) b_{2}[\Theta(\theta, \phi), \Phi(\theta, \phi)] d \Omega,
\end{aligned}
$$

where $R_{2}^{\prime}$ is the distance from the bubble cloud center to the receiver; $S_{v}$ the volume scattering coefficient of bubbles; $\Delta R$ the vertical thickness of the ensonified volume; $b_{1}, b_{2}$ are the intensity directivity functions of the transducers; and $\mathrm{Att}_{2}$ is the corresponding attenuation. The beam angles for the receiver, $\Theta$ and $\Phi$, are functions of the transducer beam angles, $\theta$ and $\phi$. The vertical thickness of the scattering volume in the bistatic case is now given by

$$
\Delta R=\frac{c \tau}{1+\cos \beta},
$$

where $\beta$ is the angle between $R_{2}^{\prime}$ and the vertical, $c$ is the sound speed, and $\tau$ is the pulse length. If $\beta$ is zero, then $\Delta R=0.5 c \tau$. In other words, the ensonified cell is elongated in the bistatic case.

The integral in (C6) is often referred to as the integrated beam pattern and can be readily calculated for given beam patterns. As mentioned above, the sidescan hydrophone beam is wide in elevation $\left(\sim 50^{\circ}\right)$ and thus the beam pattern can be considered uniform in elevation for the relatively small insonified volume of interest. In azimuth, the beam is very narrow and may be approximated by a small window of width $\delta \phi_{0} / 2 \pi$, where $\phi_{0}$ $\sim 2^{\circ}$. The source is a circular piston transducer with a $-3 \mathrm{~dB}$ two-side beam width of $3.5^{\circ}$. Thus the integrated beam pattern can be approximated as

$$
\begin{aligned}
\Psi_{D} & =\int b_{1}(\theta, \phi) b_{2}[\Theta(\theta, \phi), \Phi(\theta, \phi)] d \Omega \\
& =\frac{\delta \phi_{0}}{2 \pi} \int b_{1}(\theta, \phi) d \Omega \approx \frac{2 \delta \phi_{0}}{(k a)^{2}}
\end{aligned}
$$

for $k a \gg 1$, where $a$ is the radius of the transducer. The volume scattering coefficient and the attenuation factors are determined by the size and spatial distribution of bubbles, for which we use the results given in (B1). 
In general, the excess attenuation due to bubbles is expressed as the averaged extinction cross section of bubbles $\alpha_{e}(l)$, which may vary over the path of sound propagation. Signal intensity attenuation can be expressed as

$$
P^{2}=P_{0}^{2} \exp \left\{-\int_{0}^{L} \alpha_{e}(l) d l\right\},
$$

where the integration is over the path of propagation (absorption by seawater is negligible compared to bubble attenuation). As a first-order approximation, we consider the bubble density to be horizontally homogenous, decaying exponentially downward from the surface as in (B1). Under this assumption, it can be shown that

$$
\begin{array}{r}
\frac{\mathrm{Att}_{1}}{\mathrm{Att}} \approx \exp \left\{-\alpha_{0} D\left(\frac{2}{\cos \theta_{1}}-\exp \left(\frac{-z_{0}}{D}\right)\right.\right. \\
\left.\left.-\frac{\exp \left(-z_{0} / D\right)}{\cos \theta_{2}}\right)\right\},
\end{array}
$$

where $\alpha_{0}$ is the extinction cross section at the surface and $z_{0}$ is the depth of the scattering bubble cloud (for the angles, see Fig. 10).

We now compare (C1) with (C6), taking into account (C10) and calculate a sidelobe reduction of $18 \mathrm{~dB}$. Note that the sidelobe reflection arrives at the receiver at the same time as bistatic scatter from bubbles about $3 \mathrm{~m}$ below the surface. For the lowest sea state $(W=7.3 \mathrm{~m}$ $\mathrm{s}^{-1}$ ), the sidelobe reflection is $38 \mathrm{~dB}$ stronger than the bistatic scatter from bubble clouds. In this case, we cannot obtain any meaningful measurements of current velocity at $3-\mathrm{m}$ depth. In the highest sea state $(W=17$ $\mathrm{m} \mathrm{s}^{-1}$ ) however, the bistatic scatter is $21 \mathrm{~dB}$ stronger, and thus the sidelobe reflection has little effect. It is found that both are comparable at a wind speed of 14$15 \mathrm{~m} \mathrm{~s}^{-1}$; as the wind speed exceeds $15 \mathrm{~m} \mathrm{~s}^{-1}$, sidelobe reflection rapidly becomes insignificant. We note that acoustic attenuation due to bubbles plays a dominant role in limiting the sidelobe effect at high wind speeds. Sidelobe reflections from the surface must travel through bubble clouds, becoming progressively attenuated along the path.

\section{REFERENCES}

Agrawal, Y. C., E. A. Terray, M. A. Donelan, P. A. Hwang, A. J. Williams III, W. M. Drennan, K. K. Kahma, and S. A. Kitai- gorodskii, 1992: Enhanced dissipation of kinetic energy beneath surface waves. Nature, 359, 219-220.

Brumley, B. H., R. G. Cabrera, K. L. Deines, and E. A. Terray, 1991: Performance of a broad-band acoustic Doppler current profiler. IEEE J. Oceanic Eng., OE-16, 402-407.

Cabrera, R. G., K. Deines, B. Brumley, and E. Terray, 1987: Development of a practical coherent acoustic Doppler current profiler. Proc. Oceans '87, Halifax, NS, Canada, IEEE Oceanic Engineering Society, 93-97.

Cox, C. S., and W. Munk, 1954: Statistics of the sea surface derived from sun glitter. J. Mar. Res., 13, 198-227.

Dahl, P. H., 1999: On bistatic sea surface scattering: Field measurements and modeling. J. Acoust. Soc. Amer., 105, 2155-2169.

Dal, E. G., 1997: Acoustic Determination of Wave Kinematics in the Field. Monogr. 1, LICengineering A/S, Hellerup, Denmark, 232 pp.

Dean, R. G., and R. A. Dalrymple, 1984: Water Wave Mechanics for Engineers and Scientists. Prentice Hall, 353 pp.

Drennan, W. M., M. A. Donelan, E. A. Terray, and K. B. Katsaros, 1996: Oceanic turbulence dissipation measurements in SWADE. J. Phys. Oceanogr., 26, 808-815.

Farmer, D. M., and M. Li, 1995: Patterns of bubble clouds organized by Langmuir circulation. J. Phys. Oceanogr., 25, 1426-1440.

_, S. Vagle, and A. D. Booth, 1998: A free-flooding acoustical resonator for measurement of bubble size distributions. J. Atmos. Oceanic Technol., 15, 1132-1146.

Gemmrich, J. R., and D. M. Farmer, 1999: Near surface turbulence and thermal structure in a wind-driven sea. J. Phys. Oceanogr., 29, 480-499.

Gimenez, M. H., C. R. Sanchez-Carratala, and J. R. Medina, 1994: Analysis of false waves in numerical sea simulations. Ocean Eng., 21, 751-764.

Lygre, A., and H. E. Krogstad, 1986: Maximum entropy estimation of the directional distribution in ocean wave spectra. J. Phys. Oceanogr., 16, 2052-2060.

Medwin, H., and C. S. Clay, 1998: Fundamentals of Acoustical Oceanography. Academic Press, 712 pp.

Miller, K. S., and M. M. Rochwarger, 1972: A covariance approach to spectral moment estimation, IEEE Trans. Inf. Theory, IT-18, 588-596.

Ogilvy, J. A., 1992: Theory of Wave Scattering from Random Rough Surfaces. IOP Publishing, $277 \mathrm{pp}$.

Pinkel, R., and J. A. Smith, 1992: Repeat sequence coding for improved precision of Doppler sonar and sodar. J. Atmos. Oceanic Technol., 9, 149-163.

Pires-Silva, A. A., and J. R. Medina, 1994: False waves in wave records. Ocean Eng., 21, 765-770.

Theriault, K. B., 1986: Incoherent multibeam Doppler current profiler performance, Part I: Estimate variance. IEEE J. Oceanic Eng., OE-11, 7-15.

Trevorrow, M., and D. M. Farmer, 1992: A note on the use of Barker codes in Doppler sonar measurements. J. Atmos. Oceanic Technol., 9, 699-704.

Wurman, J., S. Heckman, and D. Boccippio, 1993: A bistatic multipleDoppler network. J. Appl. Meteor., 32, 1802-1814. 